\title{
Posits as an alternative to floats for weather and climate models
}

\author{
Milan Klöwer \\ Atmospheric, Oceanic and Planetary \\ Physics, University of Oxford \\ Oxford, UK \\ milan.kloewer@physics.ox.ac.uk
}

\author{
Peter D. Düben \\ European Centre for Medium-Range \\ Weather Forecasts \\ Reading, UK \\ peter.dueben@ecmwf.int
}

\author{
Tim N. Palmer \\ Atmospheric, Oceanic and Planetary \\ Physics, University of Oxford \\ Oxford, UK \\ tim.palmer@physics.ox.ac.uk
}

\begin{abstract}
Posit numbers, a recently proposed alternative to floating-point numbers, claim to have smaller arithmetic rounding errors in many applications. By studying weather and climate models of low and medium complexity (the Lorenz system and a shallow water model) we present benefits of posits compared to floats at 16 bit. As a standardised posit processor does not exist yet, we emulate posit arithmetic on a conventional CPU. Using a shallow water model, forecasts based on 16-bit posits with 1 or 2 exponent bits are clearly more accurate than half precision floats. We therefore propose 16 bit with 2 exponent bits as a standard posit format, as its wide dynamic range of 32 orders of magnitude provides a great potential for many weather and climate models. Although the focus is on geophysical fluid simulations, the results are also meaningful and promising for reduced precision posit arithmetic in the wider field of computational fluid dynamics.
\end{abstract}

\section{CCS CONCEPTS}

- Applied computing $\rightarrow$ Environmental sciences; Physics;

\section{KEYWORDS}

Computational fluid dynamics, weather forecast, climate projections, reduced precision, posits, floating point, computer arithmetic

\section{ACM Reference Format:}

Milan Klöwer, Peter D. Düben, and Tim N. Palmer. 2019. Posits as an alternative to floats for weather and climate models. In Conference for Next Generation Arithmetic 2019 (CoNGA'19), March 13-14, 2019, Singapore, Singapore. ACM, New York, NY, USA, 8 pages. https://doi.org/10.1145/3316279.3316281

\section{INTRODUCTION}

Weather and climate models provide predictions that are of great importance for society and economy. The Earth's climate system remains very difficult to predict even with the computational resources of the world's largest supercomputers, due to its complexity and non-linear dynamics that couple all features from the smallest time and length-scales to the largest. The forecast error of a weather forecast model has several origins [23, 24]: (i) Initial and boundary condition errors, which result from observations, data assimilation and external factors; (ii) model error, i.e. the difference

Permission to make digital or hard copies of part or all of this work for personal or classroom use is granted without fee provided that copies are not made or distributed for profit or commercial advantage and that copies bear this notice and the full citation on the first page. Copyrights for third-party components of this work must be honored.

For all other uses, contact the owner/author(s).

CoNGA'19, March 13-14, 2019, Singapore, Singapore

(C) 2019 Copyright held by the owner/author(s).

ACM ISBN 978-1-4503-7139-1/19/03.

https://doi.org/10.1145/3316279.3316281 between the mathematical model and the real world; (iii) discretisation error resulting from a finite spatial and temporal resolution of the discretised equations and (iv) rounding errors with finite precision arithmetic. In general, the forecast error is dominated by (i-iii), depending on the forecast variable and the forecast lead time. In contrast, rounding errors are usually negligible with the IEEE 754 standard on 64 bit double precision floating-point numbers [18], which is still the standard for the majority of operational weather forecasts and in climate models.

Research on reduced precision floating-point arithmetics is motivated by the potential for faster processing and communication between different elements of the computing architecture. The gained speed can be traded for increased complexity of simulations, resulting in more accurate predictions of weather and climate. The Integrated Forecast System at the European Centre for MediumRange Weather Forecasts can be run almost entirely at single precision (32 bit) without a decrease in forecast skill [33] but in $60 \%$ of the run-time. Similar progress was made at MeteoSwiss with their weather forecast model COSMO [25].

The recent boom of deep learning techniques, that require low numerical precision but high computational performance, will influence hardware development to offer more flexibility for the use of reduced numerical precision. Using simplistic chaotic models, it was shown that the majority of 64 bits at double precision do not contain real information [19]. Running algorithms used for weather forecast models at precision lower than single, for example with half precision 16-bit floats, is an active field of research, but remains challenging $[9,10,17,30]$. Most research on reduced precision modelling for weather and climate applications makes use of software emulators [8] that provide other arithmetics than the widely supported single and double precision floats. This comes with the disadvantage that simulations are orders of magnitude slower. However, software emulation allows a scientific evaluation of the use of reduced numerical precision for weather and climate simulations with no need to port the models to special hardware (such as FPGAs, [26]).

Posit numbers are a recently proposed alternative to floats and claim to provide more precision in arithmetic calculations with fewer bits in algorithms of linear algebra or machine learning [16]. However, posits remain untested for weather and climate simulations. This study therefore focuses on posit arithmetic as an alternative to floating-point arithmetic at the appealing size of 16 bit for weather and climate models. We use a Julia-based emulator on a conventional CPU, as posit hardware is not yet available. Posit research currently focuses on hardware implementations [6, 7, 12, 32].

The study is structured as follows: Section 2 introduces the posit number format and the concept of decimal precision. We analyse the dynamics of a chaotic model at low complexity with posit 
arithmetic using the Lorenz 1963 system in section 3. In section 4 we evaluate posit arithmetic in the shallow water equations, a two dimensional fluid circulation model. Section 5 discusses the results and summarises the conclusions.

\section{POSIT NUMBERS}

\subsection{The posit number format}

Following the IEEE standard on floating-point arithmetic [18], floats encode a real number in terms of a sign bit, and a fixed number of exponent and significant bits (16-bit half precision floats have 1 sign, 5 exponent and 10 significant bits). Consequently, they have a constant number of significant digits throughout their dynamic range of representable numbers. This is in contrast to posit numbers, which extend floating-point arithmetic by introducing regime bits, that are mostly responsible for the dynamic range of representable numbers. Instead of having a fixed length, regime bits are defined as the sequence of identical bits after the sign bit, which are eventually terminated by an opposite bit. The flexible length allows the significand (or mantissa) to occupy more bits when less regime bits are needed, which is the case for numbers around one. A resulting higher precision around one is traded against a gradually lower precision for very large or very small numbers. A positive posit number $p$ is decoded as $[15,16]$ (negative posit numbers are converted first to their two's complement, see Eq. 3)

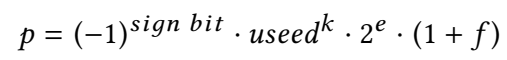

where $k$ is the number of regime bits. $e$ is the integer represented by the exponent bits and $f$ is the fraction which is encoded in the fraction (or significant) bits. The base useed $=2^{2^{e_{s}}}$ is determined by the number of exponent bits $e_{s}$. More exponent bits increase - by increasing useed - the dynamic range of representable numbers for the cost of precision. The exponent bits themselves do not affect the dynamic range by changing the value of $2^{e}$ in Eq. 1 . They fill gaps of powers of 2 spanned by useed $=4,16,256, \ldots$ for $e_{s}=1,2,3, \ldots$, and every posit number can be written as $p= \pm 2^{n} \cdot(1+f)$ with a given integer $n[7,16]$. Throughout this article we will use a notation where $\operatorname{Posit}\left(n, e_{s}\right)$ defines the posit numbers with $n$ bits including $e_{s}$ exponent bits. A posit example is provided in the Posit $(8,1)$-system (i.e. useed $=4$ )

$$
\begin{aligned}
57 & \approx 01110111_{\text {Posit }(8,1)} \\
& =(-1)^{0} \cdot 4^{2} \cdot 2^{1} \cdot\left(1+2^{-1}+2^{-2}\right)=56
\end{aligned}
$$

The sign bit is given in red, regime bits in orange, the terminating regime bit in brown, the exponent bit in blue and the fraction bits in black. The $k$-value is inferred from the number of regime bits, that are counted as negative for the bits being 0 , and positive, but subtract 1 , for the bits being 1 . The exponent bits are interpreted as unsigned integer and the fraction bits follow the IEEE floatingpoint standard for significant bits. For negative numbers, i.e. the sign bit being 1, all other bits are first converted to their two's complement (denoted with an underscore subscript) by flipping all bits and adding 1 ,

$$
\begin{aligned}
-0.28 & \approx 11011110_{\operatorname{Posit}(8,1)}=10100010_{-} \\
& =(-1)^{1} \cdot 4^{-1} \cdot 2^{0} \cdot\left(1+2^{-3}\right)=-0.28125 .
\end{aligned}
$$

After the conversion to the two's complement, the bits are interpreted in the same way as in Eq. 2.

Furthermore, posits also come with a no overflow/no underflowrounding mode: Where floats overflow and return infinity when the exact result of an arithmetic operation is larger than the largest representable number (maxpos), posit arithmetic simply returns maxpos instead, and similarly for underflow where the smallest representable number minpos is returned. This is motivated as rounding to infinity returns a result that is infinitely less correct than maxpos, although often desired to indicate that an overflow occurred in the simulation. Instead, it is proposed to perform overflowlike checks on the software level to simplify exception handling on hardware [15].

The posit number framework also highly recommends quires, an additional register on hardware to store intermediate results. Fused operations like multiply-add can therefore be executed with a single rounding error without the rounding of intermediate results. The quire concept could also be applied to floating-point arithmetic, but is technically difficult to implement on hardware as the required registers would need to be much larger in size. For fair comparison we do not take quires into account. Appendix B includes a short discussion on the benefits of quires. The posit number format is explained in more detail in Gustafson [15].

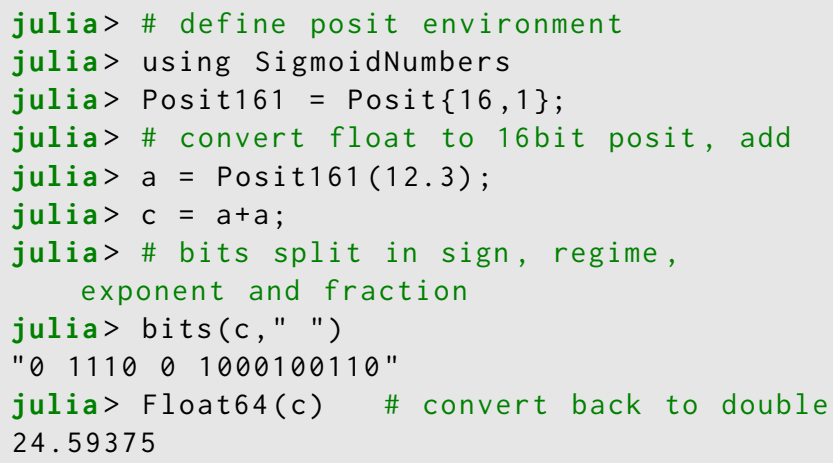

Figure 1: Example use of the posit emulator SigmoidNumbers in the Julia shell.

In order to use posits on a conventional CPU we use the posit emulator SigmoidNumbers written by Isaac Yonemoto in Julia [4]. This emulator defines conversion to and from floats and arithmetic operations with posits (see Fig. 1 for an example). Consequently, posit arithmetic can be used for the numerical integration of the Lorenz equations (section 3) and the shallow water model (section $4)$.

\subsection{Decimal precision}

The decimal precision is defined as $[15,16]$

$$
\text { decimal precision }=-\log _{10}\left|\log _{10}\left(\frac{x_{\text {repr }}}{x_{\text {exact }}}\right)\right|
$$

where $x_{\text {exact }}$ is the exact result of an arithmetic operation and $x_{\text {repr }}$ is the representable number that $x_{\text {exact }}$ is rounded to, given a specified rounding mode. For round-to-nearest rounding mode, the 
decimal precision approaches infinity when the exact result approaches the representable number and has a minimum in between two representable numbers. This minimum defines the worst-case decimal precision, i.e. the decimal precision when the rounding error is maximised. The worst-case decimal precision is the number of decimal places that are at least correct after rounding.

Fig. 2a compares the worst-case decimal precision for various 16-bit number formats: Half precision floats, 16-bit posits with various number of exponent bits, 16-bit integers and the fixedpoint format Q6.10 (6 integer bits, 10 fraction bits). Floats have a nearly constant decimal precision of almost 4 decimal places, which decreases for the subnormal numbers towards the smallest representable number minpos. Posits, on the other hand, show an increased decimal precision for numbers around 1. Posits with 1 or 2 exponent bits also have a wider dynamic range than half precision floats, in exchange for less precision for numbers on the order of $10^{4}$ as well as $10^{-4}$. Due to the no overflow/no underflowrounding mode, the decimal precision is slightly above zero outside the dynamic range.

The decimal precision of 16-bit integers is negative infinity for any number below 0.5 (round to 0 ) and maximised for the largest representable integer $2^{15}-1=32767$. Similar conclusions hold for the fixed-point format Q6.10, as the decimal precision is shifted towards smaller numbers by a factor of $\frac{1}{2}$ for each additional fraction bit. This indicates problems for reduced precision modelling: Rescaling of the equations is desired to place many arithmetic calculations near the largest representable number, however, any result beyond will lead to disastrous results, as integer overflow usually returns a negative value following a wrap around behaviour. Flexibility regarding the dynamic range can be achieved with integer arithmetic if fixed point numbers are used [26]. However, we did not achieve convincing results with integer arithmetic for the applications in this paper (see section 3.2).

\section{LORENZ 1963 SYSTEM}

\subsection{Methods}

The Lorenz system (L63, [21]) is a chaotic attractor and serves as a simplistic model for atmospheric convection. It is an extensively studied toy model for forecast uncertainty [19-21, 29] and is used here to investigate the accumulation of rounding errors in the numerical integration of a chaotic system. The Lorenz system consists of the variables $x, y$ and $z$ that are described by the following nonlinear differential equations

$$
\begin{aligned}
& \frac{d x}{d t}=\sigma(y-x) \\
& \frac{d y}{d t}=x(\rho-z)-y \\
& \frac{d z}{d t}=x y-\beta z
\end{aligned}
$$

with the typical parameter choices $\sigma=10, \rho=28$ and $\beta=\frac{8}{3}$, that permit chaotic behaviour.

To find the optimal number representation to solve Eq. 5 requires considering the dynamic range of all intermediate calculations. It is possible to influence this dynamic range using a rescaling of the
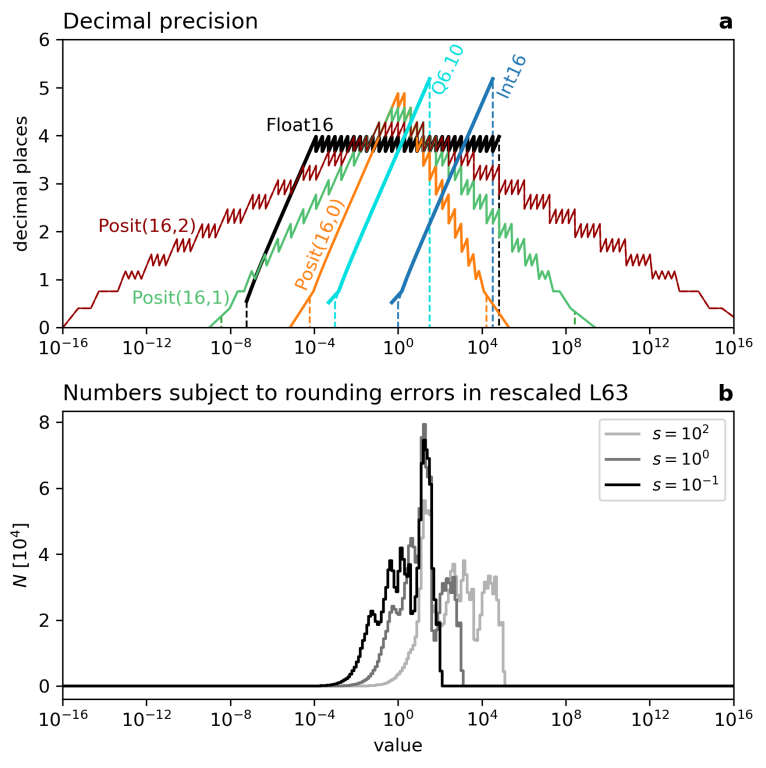

Figure 2: (a) Decimal precision of various 16-bit number formats. Dashed vertical lines indicate the dynamic range of representable numbers for each format. (b) Histogram of results of all arithmetic operations in the rescaled Lorenz system, that are subject to rounding errors, considering absolute values.

equations via a simple multiplication of the variables with a constant rescaling factor $s$. The rescaled variables are denoted as $\tilde{x}=s x$, and similarly for $\tilde{y}, \tilde{z}$. Fig. $2 \mathrm{~b}$ shows histograms for all numbers that are used to solve the Lorenz system (including intermediate calculations). A comparison to the decimal precision in Fig. 2a reveals the benefit of rescaling, especially for posit arithmetic: To profit from the increased decimal precision around 1, a scaling with $1 / 10$ is proposed to shift most calculations towards the centre of the dynamic range of representable numbers. Due to the constant decimal precision for floats, rescaling is less relevant for float arithmetic as long as no overflow nor underflow occurs. For integers, on the other hand, the Lorenz equations should be upscaled by a factor of approximately 100, to shift the range of numbers to a higher decimal precision.

We solve the equations using a fourth order Runge-Kutta method [5]. Each substep in the time integration can be written as

$$
\begin{aligned}
& \tilde{x}^{n+1}=\tilde{x}^{n}+R K_{x}\left(\tilde{y}^{n}-\tilde{x}^{n}\right) \\
& \tilde{y}^{n+1}=\tilde{y}^{n}+R K_{y}\left(\tilde{x}^{n}\left(\rho-\frac{\tilde{z}^{n}}{s}\right)-\tilde{y}^{n}\right) \\
& \tilde{z}^{n+1}=\tilde{z}^{n}+R K_{z}\left(\tilde{x}^{n} \frac{\tilde{y}^{n}}{s}-\beta \tilde{z}^{n}\right)
\end{aligned}
$$

where $R K_{x}, R K_{y}, R K_{z}$ contain the Runge-Kutta coefficient and the time step $\Delta t . R K_{x}$ also contains the parameter $\sigma$. The superscripts $n$ and $n+1$ denote the current and next substep.

The rescaling of the Lorenz system has its limitations: The nonlinear terms in Eq. 6 involve a division by the scaling constant $s$, 

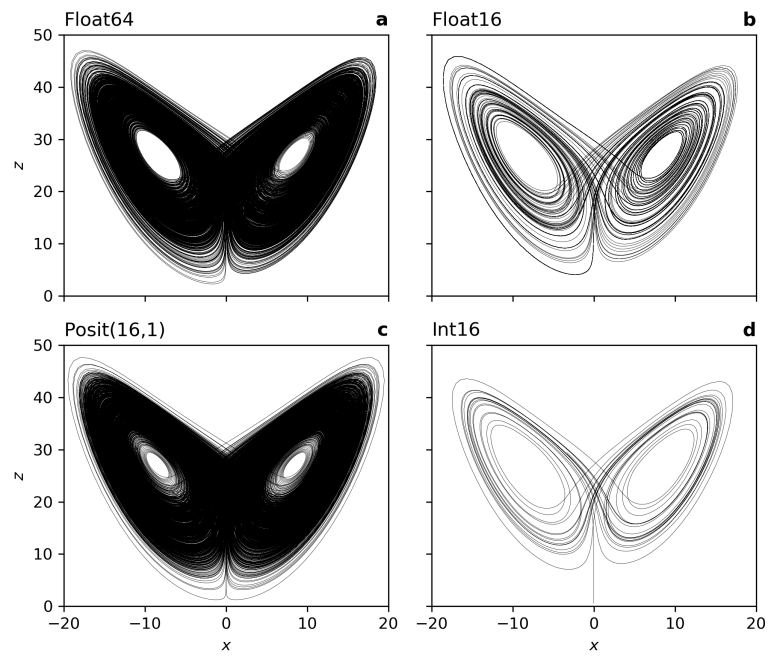

Figure 3: The Lorenz attractor computed with different arithmetics and precision. (a) 64bit double precision floats, (b) 16bit half precision floats, (c) 16-bit posits with 1 exponent bit and (d) 16-bit integers. The scaling of the Lorenz equations (Eq. 6) is (a,b) $s=1$, (c) $s=0.1$ and (d) $s=100$. All curves are integrated for the same number of time-steps.

which leads to the result of the arithmetic operations $\frac{\tilde{z}}{s}, \rho-\frac{\tilde{z}}{s}$, and $\frac{\tilde{y}}{s}$ being invariant under scaling. This is observed in the histograms of arithmetic results (Fig. 2b), as high counts of values between 1 and 50 exist for different choices of $s$. A changing shape of the histogram with $s$ is a consequence. Following these results an underlying challenge of reduced precision modelling becomes apparent: One has either to find a number format that fits the range of computed numbers, or rescale the equations to optimise their range for a given number format.

\subsection{Results}

Regardless of the initial conditions, the Lorenz system will evolve towards a set of $(x, y, z)$ points called attractor (the $\mathrm{x}, \mathrm{z}$-section of the attractor is shown in Fig. 3a). This attractor is strange, i.e. its geometric structure cannot be described in two dimensions, but is of fractal nature. While points on the model trajectory will get infinitesimally close to each other, the trajectory of the analytical Lorenz system will never repeat itself. However, if the model is discretised and if the variables are represented with finite precision, only a finite amount of distinct states can be represented and the model trajectory will necessarily repeat itself if integrated for long enough.

Integrating the Lorenz system with half precision floats yields an attractor that is repeating itself fairly early and the space that is filled by the line of the trajectory is significantly smaller when compared to the space of a trajectory with double precision (compare Fig. 3a and $b$ ). However, when using posits and a rescaling factor of $s=0.1$ the representation of the attractor is improved significantly (Fig. 3c). The results for posits looks similar to the results with 16-bit floats if no rescaling was used (not shown here).
The solution of the Lorenz system with integers fails to represent the true dynamics as the model converges to the origin (Fig. 3d).

We have calculated the so-called fractal dimension as a diagnostic to quantify the fidelity of simulations of the discretised Lorenz equations when different number formats are used. The fractal dimension quantifies how space-filling an attractor is. Using a boxcounting algorithm, we estimate the dimension of the posit attractor to be 1.78 , whereas the half precision float attractor is only 1.29 , compared to the true value of approximately $2.06[13,22]$.

\section{FLUID CIRCULATION MODEL}

\subsection{Methods}

This section will evaluate the different number formats (16-bit half precision floats, 16 -bit posits with 0,1 or 2 exponent bits) when solving the shallow water equations. The shallow water equations result from a vertical integration of the Navier-Stokes equations under the assumption that horizontal length scales are much greater than vertical scales. This assumption holds for many features of the general circulation of atmosphere and ocean $[11,31]$. The shallow water equations for the prognostic variables velocity $\mathbf{u}=(u, v)$ and sea surface elevation $\eta$ are

$$
\begin{aligned}
& \frac{\partial \mathbf{u}}{\partial t}+(\mathbf{u} \cdot \nabla) \mathbf{u}+f \hat{\mathbf{z}} \times \mathbf{u}=-g \nabla \eta+\mathbf{D}+\mathbf{F} \\
& \frac{\partial \eta}{\partial t}+\nabla \cdot(\mathbf{u} h)=0 .
\end{aligned}
$$

For the atmosphere, $\eta$ is interpreted as pressure [11]. The shallow water system is forced with a zonal wind stress $\mathrm{F}$. The dissipation term $\mathbf{D}$ removes energy on large scales (bottom friction) and on small scales (diffusion). The non-linear term $(\mathbf{u} \cdot \nabla) \mathbf{u}$ represents advection of momentum. The term $f \hat{\mathbf{z}} \times \mathbf{u}$ is the Coriolis force and $-g \nabla \eta$ is the pressure gradient force, with $g$ being the gravitational acceleration. Eq. $7 \mathrm{~b}$ is the shallow water-variant of the continuity equation, ensuring conservation of mass. The domain is a zonally periodic rectangular channel of size $2000 \mathrm{~km} \times 1000 \mathrm{~km}$, with a meridional mountain ridge in the middle of the domain. A more detailed description of the shallow water model, introducing the remaining parameters and variables in Eq. 7, is presented in appendix A. The shallow water equations are discretised using 2nd order centred finite differences on an Arakawa C-grid [2] with a grid spacing of $\Delta=20 \mathrm{~km}$ (100x50 grid points) and the Runge-Kutta fourth order method [5] is used for time integration. The advection terms are discretised using an energy and enstrophy conserving scheme [1].

To also test the use of the different number formats for the representation of passive tracers in atmosphere and ocean, we extend the shallow water equations with an advection equation. Tracers could, for example, be temperature and salinity in the ocean or aerosols in the atmosphere, which are regarded here, for simplicity, as passive (i.e. they do not influence the flow). The change of the distribution of a passive tracer $q$ that is advected by the underlying flow field is described by

$$
\frac{\partial q}{\partial t}+\mathbf{u} \cdot \nabla q=0
$$

We discretise Eq. 8 with a semi-Lagrangian advection scheme [28], which calculates the tracer concentration for a given grid cell from 
the concentration at the previous time step at a departure point, which is determined from the flow field. As the departure point is in general in between grid nodes an interpolation is required to find the concentration at the departure point. The discretisation of Eq. 8 is therefore turned into an interpolation problem.

For reduced precision it is essential to rescale the shallow water equations to avoid arithmetic operations with very large or very small results, as the dynamic range of representable numbers is limited (Fig. 2a). This is especially true for some sophisticated schemes like the biharmonic diffusion [14], which is often used to remove energy from the grid scale to ensure numerical stability. For biharmonic diffusion a fourth derivative in space is calculated. Due to the large dimension of geophysical applications, this term can get very small $O\left(10^{-20}\right)$ while viscosity coefficients are typically very large $O\left(10^{11}\right)$. The prognostic variables of Eq. 7 and 8 are typically $O\left(1 \mathrm{~ms}^{-1}\right)$ for $\mathbf{u}, O(1 \mathrm{~m})$ for $\eta$ and $O(1)$ for $q$. We can therefore retain their physical units in the discretised numerical model. However, due to the grid spacing $\Delta$ being large for geophysical flows, we need to use dimensionless Nabla operators $\tilde{\nabla}=\Delta \nabla$. The continuity equation Eq. 7 b, for example, is discretised with an explicit time integration method as

$$
\eta^{n+1}=\eta^{n}+R K_{\eta}\left(-\tilde{\nabla} \cdot(\mathbf{u} h)^{n}\right)
$$

where $R K_{\eta}$ is the Runge-Kutta coefficient times $\frac{\Delta t}{\Delta}$ which is precomputed at high precision, to avoid a division by a large value $\Delta$ and a subsequent multiplication with a large value for $\Delta t$. The other terms are rescaled accordingly $(\tilde{f}=f \Delta ; \tilde{\mathbf{F}}=\mathbf{F} \Delta$; please see appendix $\mathrm{A}$ for a discussion of the dissipation term $\mathrm{D}$ ). The entire numerical integration is performed using the various reduced precision number formats. However, posits are converted back to single precision floats for model output and some of the forcing and boundary terms that remain constant throughout the model integration are computed at higher precision during model initialisation to avoid problems with the dynamic range.

\subsection{Results}

The solution to the shallow water equations includes vigorous turbulence that dominates a meandering zonal current. Using either float or posit arithmetic with 16 bit the simulated fluid dynamics are very similar to a double precision reference: As shown in a snapshot of tracer concentration (Fig. 4) stirring and mixing can be well simulated with half precision floats and with 16-bit posits (2 exponent bits). However, the half precision simulation (Fig. 4c) deviates much faster than the posit simulation (Fig. $4 \mathrm{~b}$ ) from the double precision reference (Fig. 4a). This provides a first evidence that the accumulated rounding errors with posits are smaller than with floats. Only the posit simulations without exponent bit suffer from numerical instabilities, due to the limited dynamic range (Fig. 2a).

To quantify differences between reduced precision arithmetics we perform model forecasts that compare rounding errors. The forecast error in the shallow water model is computed as root mean square error (RMSE) taking the model based on double precision floating-point arithmetics as reference truth. We use the sea surface height (equivalent to pressure) to compute the forecast error as this variable captures the large scale circulation. The forecasts are

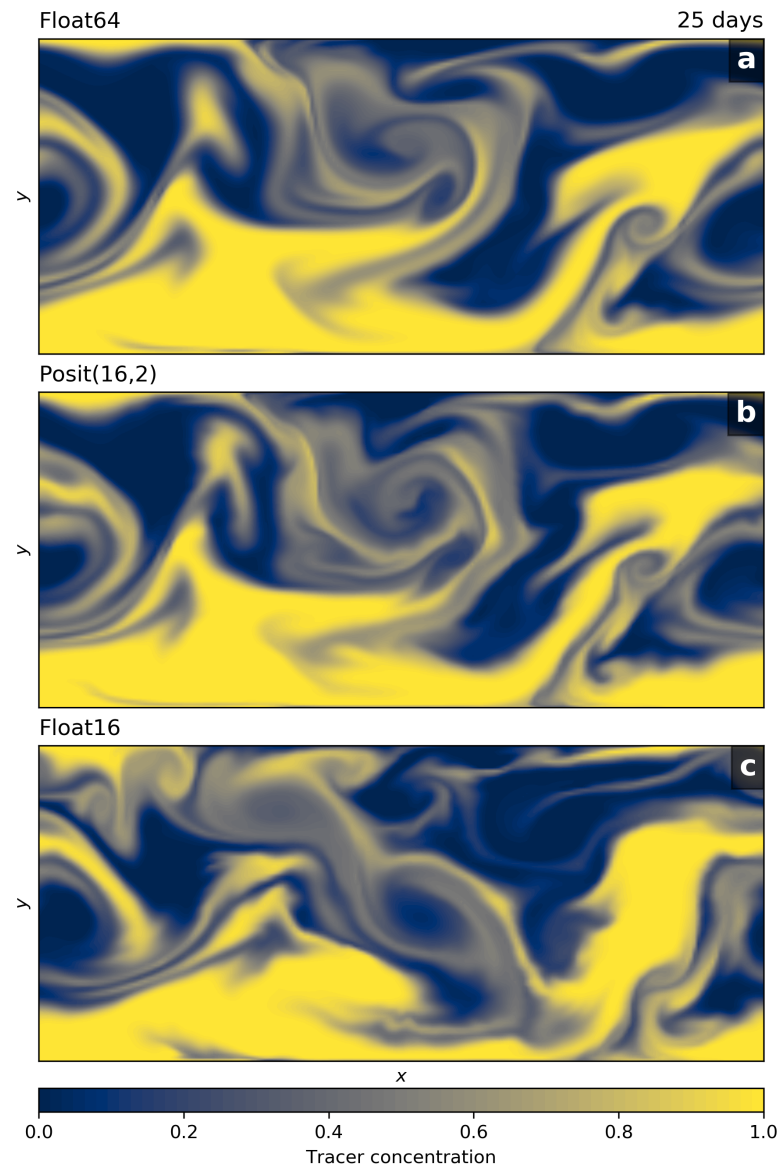

Figure 4: Snapshot of tracer concentration simulated by the shallow water model, based on (a) double precision floats and (b) posit arithmetic (16 bit with 2 exponent bits) and (c) half precision floats. The tracer was injected uniformly in the left half of the domain 25 days before. This simulation was run at a resolution of $\Delta=10 \mathrm{~km}(200 \times 100$ grid points). The corresponding video can be found at http://milank.de/ videos/swm_posit_tracer.mp4

created based on 280 different initial conditions from random start dates of a 50 year long control simulation. Each forecast is performed several times from identical initial conditions but with the various number formats. To compare the magnitude of rounding error that are caused by a reduction in precision to a realistic level of error that is caused by model discretisation, we also perform forecasts at double precision that fall back to a 3rd-order Runge-Kutta scheme for time integration and a simpler enstrophy conserving advection scheme described in Sadourny [27]. Both advection schemes have the same continuous formulation, but the Arakawa and Hsu [1] advection scheme has a wider stencil. We normalise the RMSE by the climatological mean forecast error at very long lead times. A normalised RMSE of 1 therefore means that all information of the initial conditions is removed by chaos. 


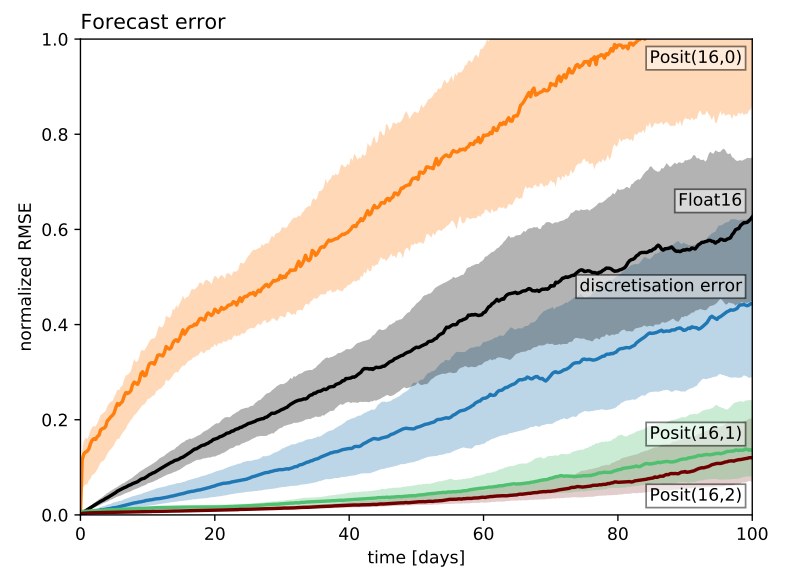

Figure 5: Forecast error measured as the root mean square error (RMSE) of sea surface height taking the double precision forecast as reference. The RMSE is normalised by a mean forecast error at very long lead times. Solid lines represent the median of $\mathbf{2 8 0}$ forecasts per number format. The shaded areas denote the interquartile range.

Clearly the best forecast is obtained for posit arithmetic with 1 or 2 exponent bits (Fig. 5), with a small accumulation of rounding errors even for lead times of 100 days. The forecast error for 16-bit posits without exponent bit increases quickly (Fig. 5), especially for short forecast lead times, but a persistence forecast, i.e. assuming the initial conditions persist over time, is still worse (not shown). Half precision floats outperform 16-bit posits without exponent bit, presumably due to the limited dynamic range of only 8 orders of magnitude compared to 12 for half precision floats (Fig. 2a). The forecast error of half precision floats is larger than the discretisation error.

\section{DISCUSSION AND CONCLUSION}

Using a software emulator we have tested posit arithmetic for weather and climate simulations. The attractor of the Lorenz 1963 model, a chaotic but simplistic model of atmospheric convection, is considerably improved using 16-bit posits with one exponent bit when compared to 16-bit half precision floats. Half precision floats can be used to perform forecasts with the shallow water model, a two-dimensional fluid circulation model that represents either atmospheric or oceanic flows. However, 16-bit posits with either 1 or 2 exponents clearly outperform floats and appear very promising for application in high performance computing for Earth System modelling. Especially 16-bit posits with 2 exponent bits, that have a wide dynamic range of 32 orders of magnitude, are likely to be widely applicable. Running computationally very demanding algorithms at 16 bit could greatly reduce the wall-clock time for weather and climate simulations on future high performance computing architecture.

The numerical discretisation that was used in this paper, with a fully-explicit time stepping scheme and 2nd order centred finite differences, is common to solve the equations of motion in fluid dynamics. However, various different methods of discretisation exist, including spectral methods, finite element/volume and implicit time stepping. The requirements on reduced precision will differ for the different algorithms and some methods may be more sensitive to rounding errors compared to the techniques that were studied in this paper. However, there is no prior reason why floats should be superior to posits in these cases and the smaller rounding errors of 16-bit posits compared to half precision floats in our applications suggest that posits are very competitive. In contrast, the wider dynamic range of posits with 1 or 2 exponent bits compared to half precision floats will facilitate the application in more complex numerical models since it will become difficult to reduce the dynamic range of all intermediate operations for complex applications. While floating-point arithmetic is then prone to overflows or underflows, posit arithmetic will be able to tolerate very small and very large numbers, although decimal precision is decreasing away from 1 for posits.

We do not show results for the bfloat16 floating-point format in this paper as rounding errors destroy the dynamics of the shallow water model. Due to the 8 exponent bits the dynamic range of bfloat16 $\left(10^{-40}\right.$ to $\left.10^{38}\right)$ remains the same as for single precision simulations. However, the small number of 7 fraction bits in this format causes rounding errors that inhibit the time evolution of the model.

In this paper, we perform model forecasts with a perfect model. Any form of model error is ignored, as the double precision reference is exactly the same model as its reduced precision counterparts. Any form of initial condition error is also ignored. Only discretisation errors are estimated by changing the advection scheme to a simpler non-energy conserving form [27] and by using a third-order instead of a fourth-order Runge-Kutta method. Here, we are likely underestimating the discretisation error of real models which also arises from the limited accuracy of spatial discretisation schemes.

This is not a realistic set-up for weather or climate models. Real models include many other sources of forecast error (see section 1) and it is likely that the contributions of rounding errors from 16-bit arithmetic would be dwarfed by errors in initial conditions or discretisation errors in many applications. As the forecast error with 16-bit posits ( 1 or 2 exponent bits) are still considerably lower than the discretisation error, this suggest that simulations with posit arithmetic of even less than 16 bit may be feasible. However, as 8 bit numbers are very likely unsuitable for applications in weather and climate models, we propose 16-bit posit with 2 exponent bits as a format that would likely meet the requirements of many algorithms used in weather and climate models. Together with a 32-bit posit format with 2 exponent bits (to match the dynamic range of single precision floats), a posit processor based on these two formats could greatly support the transition of models that are rewritten to use less than 32 bits to represent real numbers. We believe that high performance computing for Earth System modelling would benefit greatly from a processor that would support both 16 and 32-bit posit formats with 2 exponent bits.

\section{ACKNOWLEDGMENTS}

The authors would like to thank Isaac Yonemoto for providing the Julia-based emulator for posit numbers, that was used for this study. 
Milan Klöwer and Tim N. Palmer gratefully acknowledge funding by the European Research Council under grant number 741112 An Information Theoretic Approach to Improving the Reliability of Weather and Climate Simulations. Milan Klöwer is also funded by NERC grant number NE/L002612/1. Peter D. Düben gratefully acknowledges funding from the Royal Society for his University Research Fellowship as well as funding from the ESIWACE project. ESIWACE has received funding from the European Union's Horizon 2020 research and innovation program under grant agreement 675191 .

\section{SUPPLEMENTARY MATERIAL}

The shallow water model written in Julia can be found at www. github.com/milankl/juls and the corresponding video to Fig. 4 at http://milank.de/videos/swm_posit_tracer.mp4

\section{A SHALLOW WATER MODEL}

The shallow water equations are discretised on the $(x, y)$-plane over the rectangular domain $L_{x} \times L_{y}$. We associate $x$ with the zonal and $y$ with the meridional direction. The domain is centred at $30^{\circ} \mathrm{N}$ and the beta-plane approximation [31] is used to linearize the Coriolis parameter which varies linearly from $7.27 \times 10^{-5} \mathrm{~s}^{-1}$ at the southern boundary to $9.25 \times 10^{-5} \mathrm{~s}^{-1}$ at the northern boundary. The boundary conditions are periodic in zonal direction and partial slip at the northern and southern boundary. The layer thickness is $h=\eta+H(x)$, with

$$
H(x)=H_{0}-H_{1} \exp \left(-H_{\sigma}^{-2}\left(x-\frac{L_{x}}{2}\right)^{2}\right)
$$

being the undisturbed depth, representing a mountain ridge at $x=\frac{L_{x}}{2}$ spanning from the southern to the northern boundary. The standard depth is $H_{0}=500 \mathrm{~m}$. The ridge has a height of $H_{1}=50 \mathrm{~m}$. The characteristic width of the ridge is $H_{\sigma}=300 \mathrm{~km}$. The time step $\Delta t=282 \mathrm{~s}$ is chosen to resolve surface gravity waves, traveling at maximum phase speed $\sqrt{g H_{0}}$ with CFL number being 1 and gravitational acceleration $g=10 \mathrm{~ms}^{-1}$. The wind stress forcing $\mathbf{F}=\left(F_{X}, 0\right)$ is constant in time, acts only on the zonal momentum budget

$$
F x=\frac{F_{0}}{\rho h} \cos \left(\pi\left(y L_{y}{ }^{-1}-1\right)\right)^{2}
$$

and vanishes at the boundaries. The water density is $\rho=1000 \mathrm{~kg} \mathrm{~m}^{-3}$ and $F_{0}=0.12 \mathrm{~Pa}$. The dissipation term $\mathrm{D}$ is the sum

$$
\mathbf{D}=-r \mathbf{u}-v \nabla^{4} \mathbf{u}
$$

of a linear bottom drag with time scale $r^{-1}=300$ days $\approx 2.6 \times 10^{7} \mathrm{~s}$ [3] and a biharmonic diffusion with viscosity coefficient $v \approx 1.33 \times$ $10^{11} \mathrm{~m}^{4} \mathrm{~s}^{-1}$ [14]. To avoid division and subsequent multiplication with large numbers throughout the numerical model integration, we use instead

$$
\tilde{\mathbf{D}}=-\tilde{r} \mathbf{u}-\tilde{v} \tilde{\nabla}^{4} \mathbf{u}
$$

with $\tilde{r}=r \Delta \approx 0.0008 \mathrm{~ms}^{-1}$, and $\tilde{v}=v \Delta^{-3} \approx 0.16 \mathrm{~ms}^{-1}$. Computing the term $\tilde{\mathbf{D}}$ instead of $\mathbf{D}$ is required to avoid arithmetic under and overflow with floats or huge rounding errors with posit arithmetic.

The semi-Lagrangian advection scheme involves the computation of a departure point $\mathbf{x}_{d}$. In order to avoid large numbers of the coordinates $\left(L_{x}=2 \cdot 10^{6} \mathrm{~m}\right)$, we use instead a non-dimensional coordinate $\tilde{\mathbf{x}}=\mathbf{x} \Delta^{-1}$ to compute the departure point as

$$
\tilde{\mathbf{x}}_{d}=\tilde{\mathbf{x}}_{a}-\mathbf{u}_{i} \frac{\Delta t_{\mathrm{adv}}}{\Delta}
$$

where $\tilde{\mathbf{x}}_{a}$ are the coordinates of the arrival grid node and $\mathbf{u}_{i}$ the velocity interpolated on the mid-point in space and time[28]. The advective time step $\Delta t_{\mathrm{adv}}$ is much larger than $\Delta t$ to reduce numerical diffusion of the tracer due to a smaller number of interpolations. In the simulations of Fig. $4(\Delta=10 \mathrm{~km})$ the rescaled time step is $\frac{\Delta t_{\mathrm{adv}}}{\Delta} \approx \frac{2 \cdot 10^{4} \mathrm{~s}}{10^{4} \mathrm{~m}}=2 \mathrm{sm}^{-1}$ and therefore precomputed.

\section{B PERSPECTIVES FOR QUIRES}

Although we do not use quires throughout the simulations, for completeness we want to discuss computations that could greatly benefit from the use of quires. Summing the tendencies of the righthand side of Eq. 7a involves computations like

$$
u^{n+1}=u^{n}+R K_{u}\left(Q h v+\partial_{x} p+D_{x}+F_{x}\right)
$$

where $R K_{u}$ is a constant that includes the Runge-Kutta coefficient and the time step. $Q h v$ is the advection of potential vorticity, $\partial_{x} p$ is the gradient of the Bernoulli potential, $D_{x}$ is the $u$-component of bottom friction and diffusion and $F_{x}$ is the wind forcing. It is a priori not clear which of the terms $u^{n}, Q h v, \partial_{x} p, D_{x}$ or $F_{x}$ dominate the sum. Physically speaking, the shallow water model is often close to geostrophic balance, which means that the Coriolis term (which is included in $Q h v$ ) and the pressure gradient term (which is included in $\partial_{x} p$ ) oppose each other. In general, however, the dominating balance will vary in space and time and therefore it is only clear at runtime what the preferred order of addition is, which is crucial to reducing the rounding error. Using quires could solve this problem since quires will be able to reduce rounding errors greatly when performing intermediate operations. Quires may allow to perform the sum over different terms to calculate the right-hand side of the equations with a single rounding error due to 16-bit arithmetic when the final result for the new velocity value is stored.

\section{REFERENCES}

[1] Akio Arakawa and Yueh-Jiuan G Hsu. 1990. Energy Conserving and PotentialEnstrophy Dissipating Schemes for the Shallow Water Equations. , 19601969 pages. https://doi.org/10.1175/1520-0493(1990)118<1960:ECAPED>2.0.CO;

[2] Akio Arakawa and V R Lamb. 1977. Computational design of the basic dynamical processes of the UCLA general circulation model. Methods of Computational Physics 17 (1977), 173-265.

[3] Brian K. Arbic and Robert B. Scott. 2008. On Quadratic Bottom Drag, Geostrophic Turbulence, and Oceanic Mesoscale Eddies. Fournal of Physical Oceanography 38, 1 (2008), 84-103. https://doi.org/10.1175/2007JPO3653.1

[4] Jeff Bezanson, Alan Edelman, Stefan Karpinski, and Viral B. Shah. 2014. Julia: A Fresh Approach to Numerical Computing. 59, 1 (2014), 65-98. https://doi.org/ 10.1137/141000671 arXiv:1411.1607

[5] J C Butcher. 2008. Numerical Methods for Ordinary Differential Equations (2nd ed.). Wiley.

[6] Rohit Chaurasiya, John Gustafson, Rahul Shrestha, Jonathan Neudorfer, Sangeeth Nambiar, and Kaustav Niyogi. 2018. Parameterized Posit Arithmetic Hardware Generator. (2018), 9. https://posithub.org/docs/iccd_submission_v1.pdf

[7] Jianyu Chen and H Peter Hofstee. 2018. A Matrix-Multiply Unit for Posits in Reconfigurable Logic Leveraging ( Open ) CAPI. (2018), 1-5. https://doi.org/10. 1145/3190339.3190340

[8] Andrew Dawson and Peter D. Düben. 2017. Rpe v5: An emulator for reduced floating-point precision in large numerical simulations. Geoscientific Model Development 10, 6 (2017), 2221-2230. https://doi.org/10.5194/gmd-10-2221-2017

[9] Peter D. Düben. 2018. A new number format for ensemble simulations. Fournal of Advances in Modeling Earth Systems (2018). https://doi.org/10.1029/ 2018MS001420 
[10] Peter D. Düben and T. N. Palmer. 2014. Benchmark Tests for Numerical Weather Forecasts on Inexact Hardware. Monthly Weather Review 142, 10 (2014), 38093829. https://doi.org/10.1175/MWR-D-14-00110.1

[11] A E Gill. 1982. Atmosphere-Ocean Dynamics. Academic Press. 662 pages.

[12] Florian Glaser, Stefan Mach, Abbas Rahimi, Frank K. Gürkaynak, Qiuting Huang, and Luca Benini. 2017. An 826 MOPS, $210 \mathrm{uW} / \mathrm{MHz}$ Unum ALU in $65 \mathrm{~nm}$. (2017) https://doi.org/10.1109/ISCAS.2018.8351546 arXiv:1712.01021

[13] Peter Grassberger and Itamar Procaccia. 1983. Measuring the strangeness of strange attractors. Physica D: Nonlinear Phenomena 9, 1-2 (1983), 189-208. https: //doi.org/10.1016/0167-2789(83)90298-1

[14] Stephen M Griffies and Robert Hallberg. 2000. Biharmonic Friction with a Smagorinsky-Like Viscosity for Use in Large-Scale Eddy-Permitting Ocean Models. Monthly Weather Review 128, 8 (2000), 2935-2946. https://doi.org/10.1175/ 1520-0493(2000) $128<2935$

[15] John L Gustafson. 2017. Posit Arithmetic. (2017), 137 pages. https://posithub. org/docs/Posits4.pdf

[16] John L Gustafson and Isaac Yonemoto. 2017. Beating Floating Point at its Own Game: Posit Arithmetic. Supercomputing Frontiers and Innovations 4, 2 (2017), 71-86. https://doi.org/10.14529/jsfi170206

[17] Sam Hatfield, Peter Düben, Matthew Chantry, Keiichi Kondo, Takemasa Miyoshi, and Tim Palmer. 2018. Choosing the Optimal Numerical Precision for Data Assimilation in the Presence of Model Error. Fournal of Advances in Modeling Earth Systems (2018). https://doi.org/10.1029/2018MS001341

[18] IEEE. 2008. IEEE Standard for Floating-Point Arithmetic. IEEE Std 754-2008 (aug 2008), 1-70. https://doi.org/10.1109/IEEESTD.2008.4610935

[19] Stephen Jeffress, Peter Düben, and Tim Palmer. 2017. Bitwise efficiency in chaotic models. Proceedings of the Royal Society A: Mathematical, Physical and Engineering Science 473, 2205 (2017), 20170144. https://doi.org/10.1098/rspa.2017.0144

[20] Frank Kwasniok. 2014. Enhanced regime predictability in atmospheric low-order models due to stochastic forcing. Philosophical Transactions of the Royal Society A: Mathematical, Physical and Engineering Sciences 372, 2018 (2014). https: //doi.org/10.1098/rsta.2013.0286

[21] Edward N Lorenz. 1963. Deterministic Nonperiodic Flow. Journal of the Atmospheric Sciences 20 (1963), 130-141. https://doi.org/10.1175/1520-0469(1963) $020<0130$ :DNF $>2.0 . \mathrm{CO} ; 2$ arXiv:NIHMS150003

[22] Mark J. McGuinness. 1983. The fractal dimension of the Lorenz attractor. Physics Letters A 99, 1 (1983), 5-9. https://doi.org/10.1016/0375-9601(83)90052-X

[23] Tim Palmer. 2015. Build imprecise supercomputers. Nature 526 (2015), 2-3. https://doi.org/10.1038/526032a

[24] T. N. Palmer. 2012. Towards the probabilistic Earth-system simulator: A vision for the future of climate and weather prediction. Quarterly fournal of the Royal Meteorological Society 138, 665 (2012), 841-861. https://doi.org/10.1002/qj.1923

[25] Stefan Rüdisühli, André Walser, and Oliver Fuhrer. 2013. COSMO in single precision. COSMO Newsletter (2013). http://www.cosmo-model.org/content/ model/documentation/newsLetters/newsLetter14/cnl14_09.pdf

[26] Francis P. Russell, Peter D. Düben, Xinyu Niu, Wayne Luk, and T. N. Palmer. 2017 Exploiting the chaotic behaviour of atmospheric models with reconfigurable architectures. Computer Physics Communications 221 (2017), 160-173. https //doi.org/10.1016/j.cpc.2017.08.011

[27] Robert Sadourny. 1975. The Dynamics of Finite-Difference Models of the ShallowWater Equations. , 680-689 pages. https://doi.org/10.1175/1520-0469(1975) $032<0680$ :TDOFDM $>2.0$. CO; 2

[28] Piotr K. Smolarkiewicz and Janusz A. Pudykiewicz. 1992. A Class of SemiLagrangian Approximations for Fluids. Fournal of the atmospheric sciences 49, 22 (1992), 2082-2096. https://doi.org/10.1175/1520-0469(1992)049<2082:ACOSLA> 2.0.CO;2

[29] Alexis Tantet, Valerio Lucarini, and Henk A. Dijkstra. 2018. Resonances in a Chaotic Attractor Crisis of the Lorenz Flow. Fournal of Statistical Physics 170, 3 (2018), 584-616. https://doi.org/10.1007/s10955-017-1938-0 arXiv:1705.08178

[30] Tobias Thornes, Peter Düben, and Tim Palmer. 2017. On the use of scaledependent precision in Earth System modelling. Quarterly fournal of the Roya Meteorological Society 143, 703 (2017), 897-908. https://doi.org/10.1002/qi.2974

[31] G K Vallis. 2006. Atmospheric and Ocean Fluid Dynamics. Cambridge University Press. 773 pages.

[32] Laurens van Dam. 2018. Enabling High Performance Posit Arithmetic Applications Using Hardware Acceleration. Ph.D. Dissertation. Delft University of Technology.

[33] Filip Váňa, Peter Düben, Simon Lang, Tim Palmer, Martin Leutbecher, Deborah Salmond, and Glenn Carver. 2017. Single Precision in Weather Forecasting Models: An Evaluation with the IFS. Monthly Weather Review 145, 2 (2017), 495-502. https://doi.org/10.1175/MWR-D-16-0228.1 